\title{
CORRESPONDENCE
}

\section{The epidemiological impasse}

\section{To the Editors:}

We read with interest the letter of QUANJER et al. [1] persuading the Global Initiative for Chronic Obstructive Lung Disease (GOLD) committee to abandon the fixed ratio of forced expiratory volume in $1 \mathrm{~s}$ (FEV1)/forced vital capacity (FVC) $<0.70$ in favour of the lower limit of normal (LLN).

We feel that, in the current debate, some basic epidemiological principles have been overlooked. We will briefly address these principles in an attempt to clear up the discussion.

QUANJER et al. [1] point out that numerous studies have reported a higher prevalence of chronic obstructive pulmonary disease (COPD) when using the fixed FEV1/FVC $<0.70$ criterion when compared with the LLN. They interpret this as an "overdiagnosis" of COPD [2]. However, this neither implies nor ascertains that the LLN is the superior diagnostic. Conversely, one can state that the LLN leads to "underdiagnosis" of COPD. As long as either the fixed criterion or the LLN are not compared with a gold standard of COPD (when possible, a test with specificity and sensitivity approaching 100\%), one should realise that it is impossible to label either criterion as superior. The only valid conclusion of comparative studies lacking that gold standard is that both criteria differ.

How then to solve the issue? COPD is and remains a clinical diagnosis and therefore a panel decision on its absence or presence by taking into account all relevant clinical factors, such as age, respiratory complaints, smoking history, etc., is the classical approach. The fixed FEV1/FVC $<0.70$ criterion and the LLN should subsequently be compared with that panel diagnosis of COPD and the sensitivity/specificity evaluated. Unfortunately, we are unaware of any such studies and, in the mean time, any discussion and debate about the superiority/inferiority of a particular spirometric threshold will remain unproductive.

In conclusion, with the currently available literature it is impossible to label either threshold as superior or suitable.

\section{F.A.A. Mohamed Hoesein and P. Zanen}

Division of Heart and Lungs, Dept of Respiratory Medicine, University Medical Center Utrecht, Utrecht, the Netherlands.

Correspondence: F.A.A. Mohamed Hoesein, Division of Heart and Lungs, Dept of Respiratory Medicine, University Medical Center Utrecht, HP. F.02.333 PO Box 855003508 GA Utrecht, The Netherlands. E-mail: f.a.a.mohamedhoesein@umcutrecht.nl

Statement of Interest: None declared.

\section{REFERENCES}

1 Quanjer PH, Enright PL, Miller MR, et al. The need to change the method for defining mild airway obstruction. Eur Respir J 2011; 37: 720-722.
2 Mohamed Hoesein FA, Zanen P, Lammers JW. Lower limit of normal or FEV1/FVC $<0.70$ in diagnosing COPD: an evidencebased review. Respir Med 2011; 105: 907-915.

DOI: $10.1183 / 09031936.00043111$

From the authors:

We admire the extensive chronic obstructive pulmonary disease (COPD) collaborative project COPACETIC, (COPD Pathology: Addressing Critical gaps, Early Treatment and diagnosis and Innovative Concepts) [1] currently being undertaken by F.A.A. Mohamed Hoesein and co-investigators, and appreciate their letter that highlights the difficulties in defining early COPD. It is now recognised that several distinct COPD phenotypes exist in genetically susceptible adult smokers [2]. The COPACETIC study recognises two of these phenotypes: airway obstruction (detected by spirometry) and emphysema (detected by low lung tissue density from computed tomography (CT) scans and low diffusing capacity). The statistical power of studies like COPACETIC to successfully demonstrate associations between COPD phenotypes and the genetic variants that increase the risk for each phenotype will be greatly enhanced by minimising the misclassification rate for each phenotype in study participants. Misclassification of the airway obstruction phenotype for research studies and when considering a diagnosis of COPD in individual patients in clinical settings will be greatly reduced by using the fifth percentile lower limits of the normal range for both forced expiratory volume in $1 \mathrm{~s}$ (FEV1)/forced vital capacity and for FEV1, as recommended by our open letter to the Global Initiative for Chronic Obstructive Lung Disease (GOLD) committee [3].

In 1959, the Ciba Symposium coined the term "chronic nonspecific lung disease" (CNSLD) as an umbrella term grouping chronic bronchitis, asthma, emphysema and irreversible or persistent obstructive lung disease. This term was superseded by COPD, emphasising "a consistent use of asthma, emphysema, and [COPD], but restricts the use of chronic bronchitis to those patients with chronic bronchial hypersecretion without chronic airways obstruction" [4]. The vast majority of current evidence regarding the diagnosis, clinical course and treatment of COPD is based on the severe airway obstruction phenotype with a FEV1 $<60 \%$ predicted. A vast evidence gap exists in the definition of mild or early COPD. Therefore, we risk mistreatment of individual patients when we attempt to treat mild COPD, because even in smokers with respiratory symptoms and airway obstruction on post-bronchodilator spirometry, but with an FEV1 $>60 \%$ pred, we have a low degree of confidence that they belong to the 10-15\% group of genetically susceptible smokers who will rapidly lose lung function if they continue smoking [5]. Hopefully, the COPACETIC study will follow the cohort members longitudinally to discover the baseline factors that will eventually allow clinicians 
to confidently predict which young adult smokers will develop clinically important COPD.

Meanwhile, we do not think that assembling a panel of clinicians to attempt a consensus about the diagnosis of COPD in a number of cases will be useful. Establishing a diagnosis is a means to an end: intervention. Apart from smoking cessation, which is effective at any level of FEV1, the cost-effectiveness of COPD interventions when FEV1 is $>60 \%$ pred remains very poor, and, thus, screening or case-finding for early COPD should not currently be performed [6]. We are especially concerned for older persons who are classified as having COPD stage I, as such a designation may lead to inappropriate pharmacotherapy and delays in the consideration of other diagnoses, such as heart failure, which is common in older smokers [7].

\section{P.H. Quanjer*, P.L. Enright ${ }^{\#}$ and M.R. Miller \\ *Dept of Paediatrics, and Dept of Lung Diseases, Erasmus University Medical Center, Rotterdam, the Netherlands, ${ }^{*}$ College of Public Health, The University of Arizona, Tucson, AZ, USA, and "Dept of Medicine, Queen Elizabeth Hospital, Birmingham, UK.}

Correspondence: P.H. Quanjer: pquanjer@xs4all.nl

Statement of Interest: Statements of interest for all authors of this manuscript can be found at www.erj.ersjournals.com/site/ misc/statements.xhtml

\section{REFERENCES}

1 The COPACETIC collaborative project technical description. www. copacetic-study.eu/research/about-copacetic. Date last accessed: May 18, 2011.

2 Han MLK, Agusti A, Calverley PM, et al. Chronic obstructive pulmonary disease phenotypes: the future of COPD. Am J Respir Crit Care Med 2010; 182: 598-604.

3 Quanjer PH, Enright PL, Miller MR, et al. The need to change the method for defining mild airway obstruction. Eur Respir J 2011; 37: 720-722.

4 Vermeire PA, Pride NB. A "splitting" look at chronic nonspecific lung disease (CNSLD): common features but diverse pathogenesis. Eur Respir J 1991; 4: 490-496.

5 Enright P, White P. Detecting mild COPD: don't waste resources. Prim Care Respir J 2011; 20: 6-8.

6 Qaseem A, Snow V, Shekelle P, et al. Diagnosis and management of stable chronic obstructive pulmonary disease: a clinical practice guideline from the American College of Physicians. Ann Intern Med 2007; 147: 633-638.

7 Hawkins NM, Petrie MC, Jhund PS, et al. Heart failure and chronic obstructive pulmonary disease: diagnostic pitfalls and epidemiology. Eur J Heart Fail 2009; 11: 130-139.

DOI: 10.1183/09031936.00063111

\section{GOLD and the fixed ratio}

\section{To the Editors:}

QUANJER et al. [1] point out that they, and other respiratory physiologists, wish the Global Initiative for Chronic Obstructive Lung Disease (GOLD) committee to change the spirometric criteria for diagnosing chronic obstructive pulmonary disease (COPD) from the current fixed ratio of forced expiratory volume in $1 \mathrm{~s}(\mathrm{FEV} 1)$ to forced vital capacity $(\mathrm{FVC})<0.70$ to using lower limit of normal (LLN). We will try briefly to address the concerns of QUANJER et al. [1]; concerns that they have expressed in identical terms in other places.

A diagnosis of COPD is a clinical diagnosis made in a patient presenting to his/her doctor with relevant symptoms and/or exposures. In the suggested revision of the GOLD document spirometry airflow limitation will be required for the diagnosis; until now, spirometry was merely used for "confirming the diagnosis". In the GOLD document, airflow limitation is defined as a post-bronchodilator FEV1/FVC $<0.70$ [2]. We are fully aware that more subjects will have airflow limitation when the fixed ratio is used as criterion than when LLN is applied; this is easy to demonstrate in population samples. However, we are not aware of any study showing that a clinical diagnosis of COPD is more frequently, and wrongly, made if the fixed ratio is used in a diagnostic setting instead of the LLN. Indeed, there are papers showing that subjects with FEV1/FVC $<0.70$ but above LLN have a poorer prognosis than subjects with FEV1/FVC $>0.70[3,4]$.
QUANJER et al. [1] accuse GOLD of "selling sickness". We find this suggestion absurd, as GOLD does not advocate screening spirometry, or even suggest treating asymptomatic subjects with COPD. Today's problem for COPD is not overdiagnosis and overtreatment; indeed, all evidence so far points to lack of diagnosis and undertreatment of COPD.

Obviously, the choice between the fixed ratio and LLN would be simple if one was obviously correct and the other obviously wrong. However, both have advantages and disadvantages. QUANJER et al. [1] have listed their perceived errors in using the fixed ratio. However, LLN is based on the assumption that any change in lung function over time in never-smokers is solely the result of ageing and not of ageing combined with cumulative exposure to inhaled gases other than tobacco smoke. In addition, LLN is heavily dependent on the choice of reference values, as normality is solely determined by distribution of lung function values in asymptomatic never-smokers. Particularly in developing countries, where tobacco smoking may not be the most important risk factor for COPD [5], the choice of reference population will have a major impact on LLN, and thus on probability of a diagnosis of COPD. A recent study from the European Community Respiratory Health Survey has documented the effect of early life events on the likelihood of having airflow limitation in early adulthood [6] and this also underlines the need for carefully considering the choice of correct reference values in the diagnostic process. 